\title{
Active Force Closure for Multiple Objects
}

\author{
Kensuke Harada," Makoto Kaneko, \\ and Toshio Tsuji \\ Department of Artificial Complex Systems \\ Engineering, Graduate School of Engineering, \\ Hiroshima University, 1-4-1 Kagamiyama, \\ Higashi-Hiroshima 739-8527, Japan \\ Received 1 December 2000; revised 15 November 2001; accepted \\ 14 December 2001
}

\begin{abstract}
This article discusses active force closure (AFC) for the manipulation of multiple objects. AFC for multiple objects is defined in such a way that the finger can generate an arbitrary acceleration onto a certain point of multiple objects. We define two kinds of AFC: in the first, an arbitrary acceleration can be generated onto each of the objects; in the second, an arbitrary acceleration can be generated onto the center of mass of multiple objects without changing the relative position of the objects. We show that the grasped object cannot always be manipulated arbitrarily even if the first kind of AFC is satisfied. We also show that the grasped objects are manipulated like a single rigid body if the second kind of AFC is satisfied. To explain these features of AFCs, numerical examples for the grasp of three objects are shown. ๑ 2002 Wiley Periodicals, Inc.
\end{abstract}

\section{INTRODUCTION}

A robot hand is a typical end-effector of a robot arm. A potential advantage of the utilization of a multifingered robot hand is that it can manipulate an object within the hand in addition to grasping it firmly, something a simple gripper cannot do. So far, although much research has been done on manipulation by a multi-fingered robot hand, it has been implicitly assumed that a single object is being dealt with.

Let us now consider manipulating multiple objects simultaneously. Figure 1(a) shows the case where

*To whom all correspondence should be addressed; e-mail: kharada@huis.hiroshima-u.ac.jp. a multi-fingered hand approaches and envelops two cylindrical objects with significant friction. ${ }^{1}$ For two objects making rolling contact with each other, we can expect that a multi-fingered hand can easily achieve an enveloping grasp by simply pushing two links in contact with the objects. During the lifting phase, the links and two objects behave as if they were connected by mechanical gears. Due to this mechanical property, achieving an enveloping grasp for two objects seems to be even easier than doing so for a single object, especially under significant friction. This is an example of an advantageous situation for the manipulation of multiple objects.

Regarding the manipulation of an object, the concept of force closure was proposed. ${ }^{3}$ However, two 


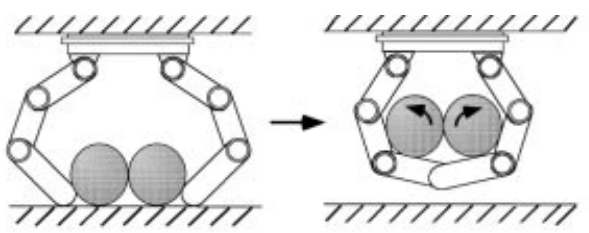

(a) Approaching and enveloping two objects

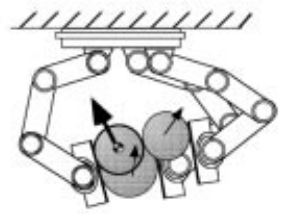

(b) The first kind of AFC

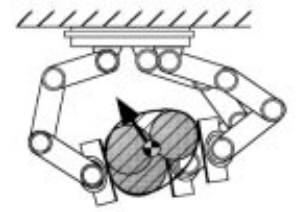

(c) The Second kind of AFC

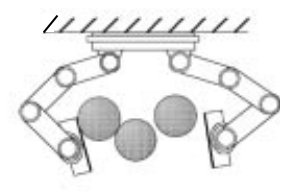

(d) Manipulation by first kind of AFC interpretations for force closure have been given: one is that "a finger can generate an arbitrary linear and angular acceleration onto the object"; and the other is that "the grasped object can structurally oppose the external force and moment without changing the joint torque." 4 For a single object, the former definition becomes the necessary and sufficient condition for manipulation, since the finger can continuously generate an arbitrary acceleration unless it is in a singular posture. On the other hand, the latter definition does not always relate to the manipulation of an object. For example, let us consider the power grasp, where each finger is allowed to have multiple contacts with an object. Although the grasped object can resist all directions of external force and moment without changing the joint torque, the object cannot be manipulated arbitrarily. To overcome this confusion, force closure was redefined and classified into active force closure (AFC) and passive force closure (PFC). ${ }^{5}$ AFC corresponds to the former definition, and PFC to the latter.

Now, let us consider extending AFC for a single object to that for multiple objects. As shown in Figure 1(b) and (c), we can define two kinds of AFC for multiple objects. As shown in Figure 1(b), the first kind of AFC focuses on one of the grasped objects at a time, and examines whether or not the robot hand can generate an arbitrary acceleration onto each one of the objects. As shown in Figure 1(d), however, when the robot hand manipulates multiple objects based on the first kind of AFC, the grasp might collapse in the next moment even if an arbitrary acceleration can be generated by the fingers in the initial phase. This is because we cannot regulate the relative motion between the objects but can only assign the direction of motion of the designated object. Therefore, for the first kind of AFC, we can see that exerting an arbitrary acceleration on an object does not always correspond to manipulating the object arbitrarily. In the second kind of AFC, we consider exerting an arbitrary acceleration at the center of mass of multiple objects, and deal with multiple objects as if they were a single rigid body, as shown in Figure 1(c). Since we must assign the desired acceleration for all objects not to cause relative motion among the objects, the second kind of AFC becomes a stronger condition for multiple-object manipulation than the first.

This article is organized as follows: After reviewing relevant previous work, we begin by showing analytical models and assumptions. We define two kinds of AFC with their characteristics, and show that determining whether each AFC is satisfied is equivalent to solving a proper linear programming problem. We further show a necessary number of fingers for satisfying each AFC. Finally, we show simulations and experiments for demonstrating each AFC.

\section{RELEVANT WORKS}

Dauchez and Delebarre ${ }^{6}$ and Kosuge, Sakai, and Kanitani ${ }^{7}$ used two manipulators holding two objects independently, and tried to apply this to an assembly task. Aiyama, Minami, and $\mathrm{Arai}^{8}$ derived the finger force for grasping multiple box-type objects with stability by utilizing the linear programming method. Mattikalli, Baraff, Khosla, and Repetto ${ }^{9}$ proposed a method for finding stable alignments of multiple objects under a gravitational field (however, they did not consider the manipulation of objects within the hand). The present authors have first studied the enveloping grasp for multiple objects. ${ }^{1}$ They have shown a condition for judging whether objects can roll at each contact point, and showed the "shovelling up" condition. The present authors also showed the basic theory of manipulating multiple objects under the kinematic constraint of rolling contact. ${ }^{2}$

As for the research on force closure, Reuleaux ${ }^{10}$ discussed force closure used in classical mechanics. Ohwovoriole ${ }^{11}$ and Salisbury and Roth ${ }^{3}$ introduced it into the research field of robotics. Mishra, Schwartz, and Sharir, ${ }^{12}$ Nguyen, $^{13}$ and other researchers ${ }^{14-16}$ 
investigated the construction of force closure grasp by a robotic hand. Kerr and Roth, ${ }^{17}$ Nakamura, Nagai, and Yoshikawa, ${ }^{18}$ and Ferrari and Canny ${ }^{19}$ discussed the optimal grasp under force closure. Force closure was introduced in books such as refs. 20 and 21, and surveyed in various papers such as refs. 16, 22, and 23. However, it has recently been pointed out by Trinkle ${ }^{4}$ that the definition of force closure includes some ambiguities. Yoshikawa ${ }^{5}$ redefined force closure and classified it into active force closure (AFC) and passive force closure (PFC). Such confusion comes about because the actuation effect caused by finger joints was not taken into consideration in the definition of force closure. Bicchi ${ }^{23}$ proposed AFC taking the finger joint into consideration. On the other hand, PFC corresponds to the concept of the power grasp. ${ }^{24,25} \mathrm{How}^{-}$ ever, there is no research dealing with the issue of force closure for multiple objects.

\section{MODELING}

Figure 2 shows the grasp of $m$ objects by $n$ fingers, where finger $j$ contacts with object $i$, and object $i$ has a common contact point with object $l$. Let $\Sigma_{R}, \Sigma_{B i}(i=$ $1, \ldots, m)$ and $\Sigma_{F j}(j=1, \ldots, n)$ be the coordinate systems fixed at the base, at the center of gravity of object $i$, and at the tip link of finger $j$, respectively. Let $\mathbf{p}_{B i} \in R^{3}$ and $\mathbf{R}_{B i} \in R^{3 \times 3}$ be the position vector and the rotation matrix of $\Sigma_{B i}$, and $\mathbf{p}_{F j} \in R^{3}$ and $\mathbf{R}_{F j} \in R^{3 \times 3}$ be those of $\Sigma_{F j}$, with respect to $\Sigma_{R}$, respectively. Let ${ }^{B i} \mathbf{p}_{C i j} \in R^{3}$ and ${ }^{F j} \mathbf{p}_{C i j} \in R^{3}$ be the position vectors of the contact point of finger $j$ with respect to $\Sigma_{B i}$ and $\Sigma_{F j}$, respectively. Also, let ${ }^{B i} \mathbf{p}_{C O t} \in R^{3}(t=1, \ldots, r)$

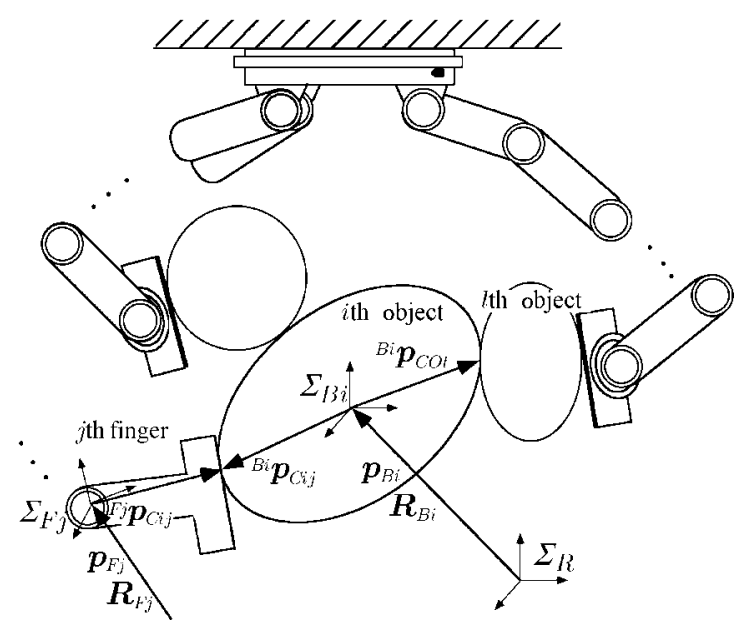

Figure 2. Model of the system. be the position vector of the common contact point between object $i$ and object $l$, with respect to $\Sigma_{B i}$. We assume that all fingers have sufficient degrees of freedom to exert an arbitrary contact force $\left(s_{j} \geq 3\right)$ where $s_{j}$ denotes the number of joints in finger $j$.

We assume that object $i$ maintains contact with finger $j$, and that object $i$ maintains contact with object $l$ at the contact point. These relationships are expressed as follows:

$$
\begin{gathered}
\mathbf{D}_{B i j}\left[\begin{array}{c}
\dot{\mathbf{p}}_{B i} \\
\boldsymbol{\omega}_{B i}
\end{array}\right]=\mathbf{D}_{F j}\left[\begin{array}{c}
\dot{\mathbf{p}}_{F j} \\
\boldsymbol{\omega}_{F j}
\end{array}\right] \\
\mathbf{D}_{O i t}\left[\begin{array}{c}
\dot{\mathbf{p}}_{B i} \\
\boldsymbol{\omega}_{B i}
\end{array}\right]=\mathbf{D}_{O l t}\left[\begin{array}{c}
\dot{\mathbf{p}}_{B l} \\
\boldsymbol{\omega}_{B l}
\end{array}\right] \\
\mathbf{D}_{B i j}=\left[\mathbf{I}_{3}-\left(\mathbf{R}_{B i}{ }^{B i} \mathbf{p}_{C i j} \times\right)\right] \in R^{3 \times 6} \\
\mathbf{D}_{F j}=\left[\mathbf{I}_{3}-\left(\mathbf{R}_{F j}{ }^{F i} \mathbf{p}_{C i j} \times\right)\right] \in R^{3 \times 6} \\
\mathbf{D}_{O i t}=\left[\mathbf{I}_{3}-\left(\mathbf{R}_{B i}{ }^{B i} \mathbf{p}_{C O t} \times\right)\right] \in R^{3 \times 6}
\end{gathered}
$$

where $\mathbf{I}_{3}$ denotes the $3 \times 3$ identity matrix, $(* \times)$ denotes the skew-symmetric matrix equivalent to the vector product, and $\boldsymbol{\omega}_{B i}$ and $\boldsymbol{\omega}_{F j}$ denote the angular velocity vectors of $\Sigma_{B i}$ and $\Sigma_{F j}$ with respect to $\Sigma_{R}$, respectively. Aggregating Eqs. (1) and (2) for $j=1, \ldots, n$ and $t=1, \ldots, l$, the equation of object motion constraint is derived as follows:

$$
\mathbf{D}_{C} \dot{\mathbf{p}}_{C F}=\mathbf{D}_{B} \dot{\mathbf{p}}_{B}
$$

where $\dot{\mathbf{p}}_{C F}=\left[\dot{\mathbf{p}}_{C F 1}^{T} \ldots \dot{\mathbf{p}}_{C F n}^{T}\right]^{T} \in R^{3 n}, \dot{\mathbf{p}}_{C F j}=\mathbf{D}_{F j}$

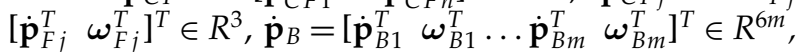
$\mathbf{D}_{C}=\left[\begin{array}{ll}\mathbf{I}_{3 n} & \mathbf{o}\end{array}\right]^{T} \in R^{(3 n+3 r) \times 3 n}, \mathbf{D}_{B}=\left[\begin{array}{ll}\mathbf{D}_{C B}^{T} & \mathbf{D}_{O}^{T}\end{array}\right]^{T} \in$ $R^{(3 n+3 r) \times 6 m} . \mathbf{D}_{C B} \in R^{3 n \times 6 m}$ has $\mathbf{D}_{B i j}$ as the element from $(3 j-2,6 i-5)$ th to $(3 j, 6 i)$ th, and $\mathbf{D}_{O} \in R^{3 r \times 6 m}$ has $\mathbf{D}_{\text {Oit }}$ as the element from $(3 t-2,6 i-5)$ th to $(3 t, 6 i)$ th, and $-\mathbf{D}_{\text {Olt }}$ as the element from $(3 t-2$, $6 l-5)$ th to $(3 t, 6 l)$ th when $i<l$. $\dot{\mathbf{p}}_{C F}$ shows the velocity of the tip link with respect to $\Sigma_{R}$, evaluated at the contact point.

\subsection{Statics}

Through the relationship of duality between force and infinitesimal displacement, we can obtain the force balance equation for multiple objects as follows:

$$
\mathbf{f}_{B}=\mathbf{D}_{B}^{T} \mathbf{f}_{C}
$$

where $\mathbf{f}_{B}=\left[\begin{array}{lllll}\mathbf{f}_{B 1}^{T} & \mathbf{n}_{B 1}^{T} & \ldots & \mathbf{f}_{B m}^{T} & \mathbf{n}_{B m}^{T}\end{array}\right]^{T} \in R^{6 m}, \mathbf{f}_{C}=$ $\left[\mathbf{f}_{C B}^{T} \mathbf{f}_{O}^{T}\right]^{T} \in R^{3 n+3 r}, \mathbf{f}_{C B}=\left[\mathbf{f}_{C 1}^{T} \ldots \mathbf{f}_{C n}^{T}\right]^{T}, \mathbf{f}_{O}=\left[\mathbf{f}_{C O 1}^{T} \ldots\right.$ $\left.\mathbf{f}_{C O r}^{T}\right]^{T}$, and $\mathbf{f}_{B i}$ and $\mathbf{n}_{B i}(i=1, \ldots, m)$ denote the force 
and the moment at the center of gravity of the object $i$, respectively. $\mathbf{f}_{C j}(j=1, \ldots, n)$ and $\mathbf{f}_{C O t}(t=1, \ldots, r)$ denote the contact force applied by finger $j$, and the contact force at the $t$ th contact between objects (where we assume that object $l$ applies contact force to object $i$ when $i<l$ ), respectively.

In order to be released from the nonlinear constraint, we approximate the friction cone by the polyhedral convex cone as follows:

$$
\mathbf{f}_{C}=\mathbf{V} \boldsymbol{\lambda}, \quad \boldsymbol{\lambda} \geq 0
$$

where

$$
\begin{aligned}
\mathbf{V} & =\left[\begin{array}{cccccc}
\mathbf{V}_{1} & \ldots & \mathbf{o} & \mathbf{o} & \ldots & \mathbf{o} \\
\vdots & \ddots & \vdots & \vdots & & \vdots \\
\mathbf{o} & \ldots & \mathbf{V}_{n} & \mathbf{o} & \ldots & \mathbf{o} \\
\mathbf{o} & \ldots & \mathbf{o} & \mathbf{V}_{O 1} & \ldots & \mathbf{o} \\
\vdots & & \vdots & \vdots & \ddots & \vdots \\
\mathbf{o} & \ldots & \mathbf{o} & \mathbf{o} & \ldots & \mathbf{V}_{O r}
\end{array}\right] \\
\mathbf{V}_{j} & =\left[\mathbf{v}_{j 1}, \ldots, \mathbf{v}_{j h}\right], \quad j=1, \ldots, n \\
\mathbf{V}_{O t} & =\left[\mathbf{v}_{O t 1}, \ldots, \mathbf{v}_{O t h}\right], \quad t=1, \ldots, r \\
\lambda & =\left[\lambda_{11}, \ldots, \lambda_{1 h}, \lambda_{21}, \ldots, \lambda_{n h}, \lambda_{O 11}, \ldots, \lambda_{O r h}\right]
\end{aligned}
$$

$\mathbf{v}_{j \kappa}$ and $\mathbf{v}_{\text {Ot }}(j=1, \ldots, n, t=1, \ldots, k, \kappa=1, \ldots, h)$ denote the span vectors of the convex polyhedral cones, and $\lambda_{j \kappa}$ and $\lambda_{O t \kappa}$ denote the magnitude of contact force along the span vectors. This approximation enables us to treat the nonlinear friction constraint as a linear one. Furthermore, we choose the span vectors so that they coincide with the boundary surface of the actual friction cone. Such an approximation of the friction cone enables us to evaluate the contact force conservatively from the viewpoint of avoiding slipping motion at the contact point.

\subsection{Dependency of Contact Force}

To confirm whether an arbitrary acceleration can be generated onto multiple objects, we must know the contact force among the objects as well as the contact force applied by each finger. For this purpose, we will now clarify the dependency of contact force among objects. ${ }^{2}$ The equation of motion of the grasped objects is given by

$$
\mathbf{M}_{B} \ddot{\mathbf{p}}_{B}+\mathbf{h}_{B}=\mathbf{D}_{C B}^{T} \mathbf{f}_{C B}+\mathbf{D}_{O}^{T} \mathbf{f}_{O}
$$

where $\mathbf{M}_{B}=\operatorname{diag}\left[m_{B 1} \mathbf{I}_{3} \mathbf{H}_{B 1} \cdots m_{B m} \mathbf{I}_{3} \mathbf{H}_{B m}\right], m_{B i}$,
$\mathbf{H}_{B i}$, and $\mathbf{h}_{B}$ denote the inertia matrix, the mass of object $i$, and the inertia tensor of object $i$, and the vector with respect to the centrifugal and the Coriolis force, respectively. From Eq. (3), the motion constraint among objects can be expressed as

$$
\mathbf{D}_{O} \dot{\mathbf{p}}_{B}=\mathbf{o}
$$

Using Eq. (6) and the differentiation of Eq. (7), the following relation is derived:

$$
\mathbf{A f _ { C }}=\mathbf{b}
$$

where $\mathbf{A}=\left[\mathbf{D}_{O} \mathbf{M}_{B}^{-1} \mathbf{D}_{C B}^{T} \mathbf{D}_{O} \mathbf{M}_{B}^{-1} \mathbf{D}_{O}^{T}\right]$ and $\mathbf{b}=$ $\mathbf{D}_{O} \mathbf{M}_{B}^{-1} \mathbf{h}_{B}-\mathbf{D}_{O} \mathbf{p}_{B}$. Eq. (8) shows the dependency of the contact force, namely that $\mathbf{f}_{O}$ is determined depending on $\mathbf{f}_{C B}$. If $\mathbf{D}_{O} \mathbf{M}_{B}^{-1} \mathbf{D}_{O}^{T}$ in Eq. (8) is nonsingular, $\mathbf{f}_{O}$ can be uniquely obtained for a given finger force. Therefore, the nonsingularity of $\mathbf{D}_{O} \mathbf{M}_{B}^{-1} \mathbf{D}_{O}^{T}$ is a condition for finding the unique $\mathbf{f}_{O}$. Since $\mathbf{M}_{B}$ is nonsingular, the nonsingularity of $\mathbf{D}_{O} \mathbf{M}_{B}^{-1} \mathbf{D}_{O}^{T}$ is equivalent to the following condition:

$$
\operatorname{rank}_{O}=3 r
$$

\section{DEFINITION OF AFCS}

We define AFC as follows:

AFC for Multiple Objects. An arbitrary translational and angular acceleration can be exerted at a reference point of multiple objects.

We define two kinds of AFCs for multiple objects, as extensions of AFC for a single object. The first kind of AFC focuses on one of the grasped objects; the second kind focuses on the center of mass of multiple objects. These AFCs are defined as follows:

The First Kind of AFC. Focusing on one of the objects, if an arbitrary acceleration can be exerted on each of the objects, the grasp is termed the first kind of AFC.

The Second Kind of AFC. Focusing on the center of mass of multiple objects, if an arbitrary acceleration can be exerted at the center of mass of multiple objects without causing relative motion among the objects, the grasp is termed the second kind of AFC.

Objects cannot always be manipulated arbitrarily, even if an arbitrary acceleration can be exerted on each object. The second kind of AFC ensures that multiple objects can be manipulated like a single object. 
Now, we formulate the conditions for satisfying each definition.

The First Kind of AFC. Assuming that all objects are stationary $\left(\dot{\mathbf{p}}_{B}=0\right)$, we focus on the center of gravity of the $i$ th object. By using Eqs. (4) and (6), the acceleration exerted by the fingers is given by:

$$
\mathbf{M}_{B} \ddot{\mathbf{p}}_{B}=\mathbf{f}_{B}
$$

By extracting the equation for the $i$ th object from Eq. (10), the following relation is derived:

$$
\mathbf{M}_{B i}\left[\begin{array}{c}
\ddot{\mathbf{p}}_{B i} \\
\dot{\boldsymbol{\omega}}_{B i}
\end{array}\right]=\left[\begin{array}{c}
\mathbf{f}_{B i} \\
\mathbf{n}_{B i}
\end{array}\right]
$$

where $\mathbf{M}_{B i}=\operatorname{diag}\left[m_{B i} \mathbf{I}_{3} \mathbf{H}_{B i}\right]$. The total force and moment of the $i$ th object is expressed as follows:

$$
\left[\begin{array}{l}
\mathbf{f}_{B i} \\
\mathbf{n}_{B i}
\end{array}\right]=\mathbf{D}_{B i}^{T} \mathbf{f}_{C}
$$

where $\mathbf{D}_{B i}^{T}$ is derived by extracting the lines of $\mathbf{D}_{B}^{T}$ from the $(6 i+1)$ th to the $(6 i+6)$ th. By using Eqs. (12), (11), (5), and (8), we can formulate a linear programming problem as follows:

Minimize

$$
z=\mathbf{a}^{T} \boldsymbol{\lambda}, \quad \mathbf{a}=[1 \cdots 1]^{T}
$$

Subject to

$$
\begin{aligned}
\mathbf{M}_{B i}\left[\begin{array}{c}
\ddot{\mathbf{p}}_{B i} \\
\dot{\boldsymbol{\omega}}_{B i}
\end{array}\right] & =\mathbf{D}_{B i}^{T} \mathbf{V} \boldsymbol{\lambda} \\
\mathbf{A V} \boldsymbol{\lambda} & =\mathbf{b} \\
\boldsymbol{\lambda} & \geq 0
\end{aligned}
$$

For a given set of accelerations, we now examine whether there exists a set of contact forces within the friction constraint. We consider twelve sets of unit accelerations, $\left[\ddot{\mathbf{p}}_{B i}^{T} \dot{\boldsymbol{\omega}}_{B i}^{T}\right]^{T}=\mathbf{e}_{1}, \mathbf{e}_{2}, \ldots, \mathbf{e}_{6},-\mathbf{e}_{1},-\mathbf{e}_{2}, \ldots$, $-\mathbf{e}_{6}$, where $\mathbf{e}_{k} \in R^{6}(k=1, \ldots, 6)$ denotes the $k$ th unit vector. Substituting these unit accelerations into a linear programming problem, if all the linear programming problems have solutions, it is guaranteed that an arbitrary acceleration can be exerted on the $i$ th object (given the proof for the AFC of a single object, which is provided in ref. 18). If all objects satisfy this condition, the grasp is termed the first kind of AFC. It should be noted that we are not interested in obtaining the optimal solution to the linear programming problem, but in confirming whether there exists a set of contact forces that make a focused object move in the desired direction.

The Second Kind of AFC. Supposing that all objects are stationary, we focus on the center of mass of multiple objects. The relation between the acceleration of the center of mass and the acceleration of each object is derived as follows:

$$
\ddot{\mathbf{p}}_{B}=\mathbf{D}_{G}\left[\begin{array}{c}
\ddot{\mathbf{p}}_{G} \\
\dot{\boldsymbol{\omega}}_{G}
\end{array}\right]
$$

where $\ddot{\mathbf{p}}_{G}$ and $\dot{\boldsymbol{\omega}}_{G}$ are the translational and angular acceleration at the center of mass of the objects, respectively, and

$\mathbf{D}_{G}=\left[\begin{array}{ccccc}\mathbf{I}_{3} & \mathbf{o} & \ldots & \mathbf{I}_{3} & \mathbf{o} \\ \left(\left(\mathbf{p}_{B 1}-\mathbf{p}_{G}\right) \times\right) & \mathbf{I}_{3} & \ldots & \left(\left(\mathbf{p}_{B m}-\mathbf{p}_{G}\right) \times\right) & \mathbf{I}_{3}\end{array}\right]^{T}$

By using Eqs. (5), (8), (10), and (14), we can formulate the following linear programming problem:

Minimize

$$
z=\mathbf{a}^{T} \boldsymbol{\lambda}, \quad \mathbf{a}=[1 \cdots 1]^{T}
$$

Subject to

$$
\begin{aligned}
\mathbf{M}_{B} \mathbf{D}_{G}\left[\begin{array}{c}
\ddot{\mathbf{p}}_{G} \\
\dot{\boldsymbol{\omega}}_{G}
\end{array}\right] & =\mathbf{D}_{B}^{T} \mathbf{V} \boldsymbol{\lambda} \\
\mathbf{A V} \boldsymbol{\lambda} & =\mathbf{b} \\
\boldsymbol{\lambda} & \geq 0
\end{aligned}
$$

Considering twelve sets of unit accelerations, $\left[\ddot{\mathbf{p}}_{G}^{T} \dot{\boldsymbol{\omega}}_{G}^{T}\right]^{T}=\mathbf{e}_{1}, \mathbf{e}_{2}, \ldots, \mathbf{e}_{6},-\mathbf{e}_{1},-\mathbf{e}_{2}, \ldots,-\mathbf{e}_{6}$, if all the corresponding linear programming problems have solutions, it is guaranteed that an arbitrary acceleration can be exerted on the center of mass of the multiple objects. Such a grasp is termed the second kind of AFC.

Note from Eq. (14) that the desired acceleration corresponds to each object corresponding to the desired acceleration at the center of mass of the objects. In that sense, relative motion among the objects does not occur in the second kind of AFC.

\section{NECESSARY NUMBER OF FINGERS}

We will now discuss the number of fingers required for AFC of multiple objects. We first consider the first kind of AFC. In the case of point contact with friction, 
it is known that for the force closure of a single object $[5,13]$, the grasp is force closure if and only if the three friction cones share a common area on the plane including the three contact points (in the 3D case), and that the line connecting the two contact points is strictly within the two friction cones (in the 2D case). By simply extending the AFC for a single object to the first kind of AFC for multiple objects, we can obtain the following condition.

Necessary Number of Fingers for First Kind of AFC. To satisfy the first kind of AFC,

$$
\begin{array}{ll}
n \geq 3 & \text { for } 3 D \\
n \geq 2 & \text { for } 2 D
\end{array}
$$

is required. We will show an example in the next section in which the first kind of AFC is satisfied for the grasp by two fingers in 2D.

To satisfy the second kind of AFC, when all the fingers are locked, the objects should have no degrees of freedom of motion. Substituting $\dot{\mathbf{p}}_{C F}=\mathbf{o}$ into Eq. (3), and considering that the obtained equation should not have a solution except for $\dot{\mathbf{p}}_{B}=\mathbf{o}$, we can assume the following condition for the second kind of AFC:

$$
\operatorname{Ker}\left(\mathbf{D}_{B}\right)=\emptyset
$$

When $\mathbf{D}_{B}$ is a full-rank matrix, Eq. (18) is equivalent to $3 n+3 r \geq 6 m$ in $3 \mathrm{D}$ and $2 n+2 r \geq 3 m$ in $2 \mathrm{D}$, since $\mathbf{D}_{B} \in R^{(3 n+3 r) \times 6 m}$ in $3 \mathrm{D}$ and $\mathbf{D}_{B} \in R^{(2 n+2 r) \times 3 m}$ in 2D. Moreover, to maintain the equilibrium, Eq. (4) should have solution satisfying $\mathbf{f}_{C} \neq \mathbf{o}$ when $\mathbf{f}_{B}=\mathbf{o}$, that is, $3 n+3 r>6 m$ in $3 \mathrm{D}$ and $2 n+2 r>3 m$ in $2 \mathrm{D}$. Therefore, we obtain the following conditions.

Necessary Number of Fingers for Second Kind of AFC. To satisfy the second kind of AFC,

$$
\begin{array}{ll}
n>2 m-r & \text { for 3D } \\
n>(3 m-2 r) / 2 & \text { for 2D }
\end{array}
$$

is required. For example, for two objects with one contact point between them $(m=2, r=1)$ in $2 \mathrm{D}$, the necessary number of fingers is three.

We note that the conditions obtained in this section are necessary conditions, since we do not consider the condition in which the contact forces are included in the friction cone.

\section{EXAMPLES}

We performed numerical examples for three grasp configurations, as shown in Figure 3. For simplicity, we consider a 2D grasp of unit discs, where the mass and the friction angle at each contact point are set as unity and $\pi / 4$, respectively.

We first examine the first kind of AFC for cases 1 and 2. Both cases satisfy the condition for necessary number of fingers, since there are two fingers. Let us focus on the object in the center of case 1 . Solving the linear programming problem (Eq. (13)), we find that it provides no solution for acceleration in a downward direction. Therefore, case 1 does not satisfy the first kind of AFC. Now, let us consider case 2. We again focus on the center object; the solutions of the linear programming problem are shown in Figure 4. Note that for a 2D model, the number of linear independent accelerations is three. Although we must solve six linear programming problems, we show the result of only three of them, since the grasp is symmetrical with respect to the object in the center. In Figure 4, the dotted lines denote the contact force corresponding to the acceleration expressed by the solid line. From the result shown in Figure 4, we can see that an arbitrary
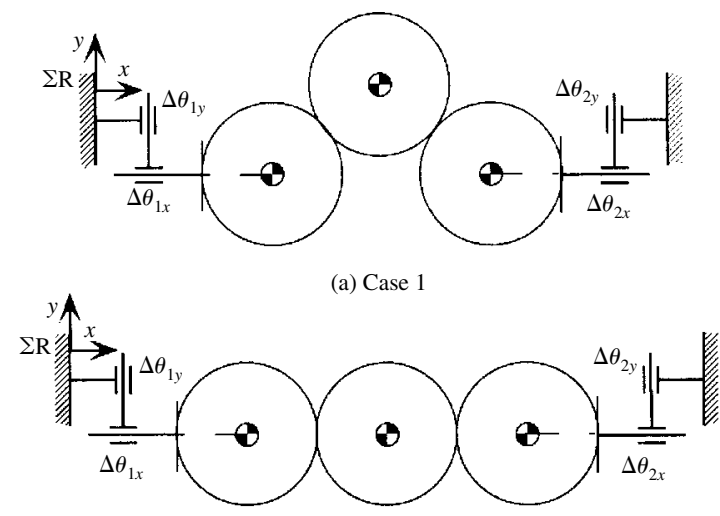

(b) Case 2

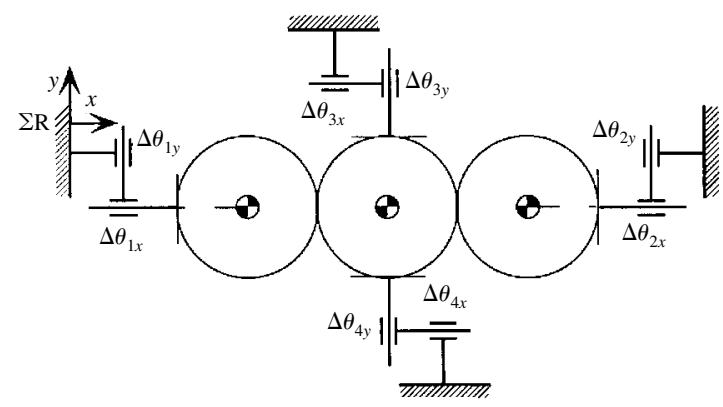

(c) Case 3

Figure 3. Numerical examples of three three-object grasps. 


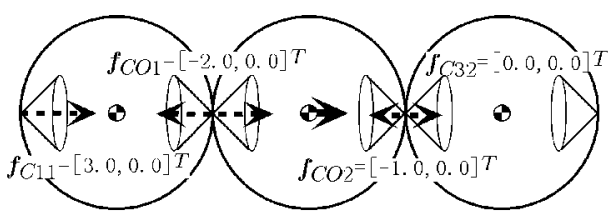

(a) $\left[\ddot{\boldsymbol{p}}_{B 2}{ }^{T} \dot{\boldsymbol{\omega}}_{B 2} T\right] T=\left[\begin{array}{lll}1 & 0 & 0\end{array}\right] T$

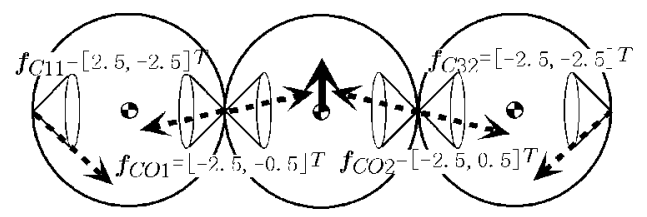

(b) $\left[\begin{array}{ll}\ddot{\boldsymbol{p}}_{B ?}{ }^{T} & \dot{\boldsymbol{\omega}}_{B^{2},}^{T}\end{array}\right]^{T}-\left[\begin{array}{lll}0 & 1 & 0\end{array}\right]^{T}$

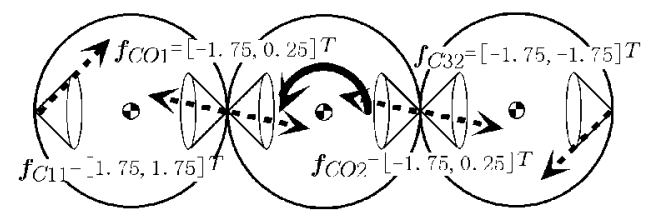

(c) $\left[\ddot{\boldsymbol{p}}_{B ?}{ }^{T} \dot{\boldsymbol{\omega}}_{B ?}{ }^{T}\right]^{T}=\left[\begin{array}{lll}0 & 0 & 1\end{array}\right]^{T}$

Figure 4. Numerical solutions (first kind of AFC for the center object).

acceleration can be exerted on the object in the center. We focus further on the left-hand object, on which an arbitrary acceleration can also be exerted, as shown in Figure 5. We note that the results for the right-hand

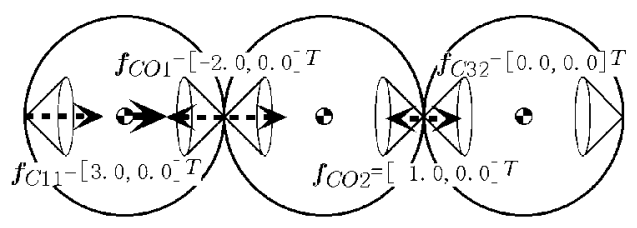

(a) $\left[\ddot{\boldsymbol{p}}_{B 1^{T}} \dot{\boldsymbol{\omega}}_{B 1}{ }^{T}\right]^{T}=\left[\begin{array}{lll}1 & 0 & 0\end{array}\right]^{T}$

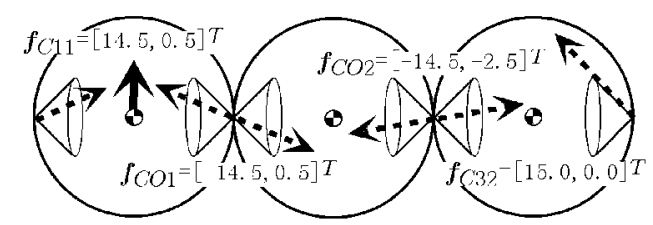

(b) $\left.\left|\ddot{p}_{B 1}^{T} \dot{\omega}_{B 1}^{T}\right|^{T}-\mid \begin{array}{lll}0 & 1 & 0\end{array}\right]^{T}$

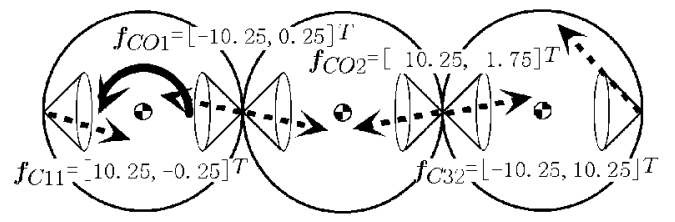

(c) $\left[\ddot{\boldsymbol{p}}_{B 1}^{T} \dot{\boldsymbol{\omega}}_{B 1}^{T}\right]^{T}=\left[\begin{array}{lll}0 & 0 & 1\end{array}\right]^{T}$

Figure 5. Numerical solutions (first kind of AFC for the left-hand object).

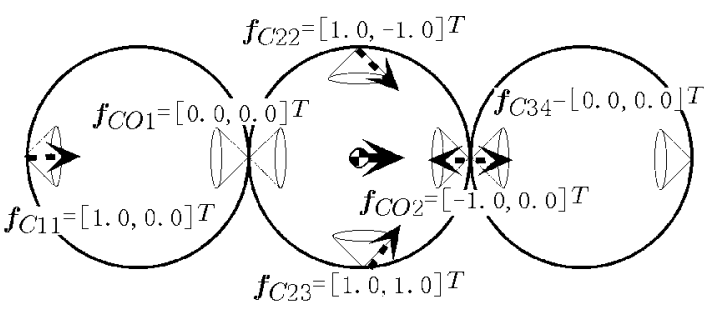

(a) $\left[\ddot{\boldsymbol{p}}_{G} T \dot{\boldsymbol{\omega}}_{G} T\right] T=\left[\begin{array}{lll}1 & 0 & 0\end{array}\right] T$

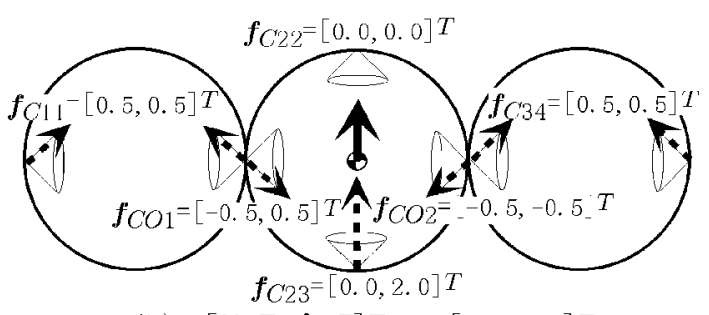

(b) $\left[\begin{array}{ll}\ddot{\boldsymbol{p}}_{G}{ }^{T} & \dot{\boldsymbol{\omega}}_{G}{ }^{T}\end{array}\right]^{T}=\left[\begin{array}{lll}0 & 1 & 0\end{array}\right]^{T}$

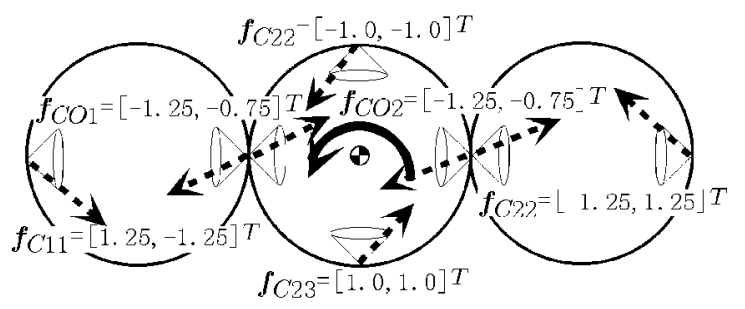

(c) $\left[\ddot{\boldsymbol{p}}_{G} T \dot{\boldsymbol{\omega}}_{G} T\right] T=\left[\begin{array}{lll}0 & 0 & 1\end{array}\right] T$

Figure 6. Numerical solutions (second kind of AFC for case 3).

object can be obtained from those for the left-hand object. Therefore, since an arbitrary acceleration can be exerted on all objects, we can see that case 2 satisfies the first kind of AFC. We confirmed that for four discs in line grasped by two fingers, the first kind of AFC can also be satisfied.

Next, let us examine the second kind of AFC by comparing case 2 and case 3 . For three objects with two contact points among them $(m=3, r=2)$, the necessary number of fingers for the second kind of AFC is four. Solving the linear programming problem (Eq. (15)), we find that case 2 does not satisfy the second kind of AFC, whereas case 3 satisfies it. The solutions of the linear programming problem (Eq. (15)) for case 3 are illustrated in Figure 6, in which we can see that the grasped objects can be manipulated without changing the relative positions.

\section{EXPERIMENT}

We performed an experiment to show the manipulation of multiple objects satisfying the AFC proposed in 
this article. In this experiment, we confirmed only the second kind of AFC, using the planar grasp of two objects by three fingers. We used the Hiroshima-hand, ${ }^{26}$ in which each finger has three links whose lengths are $l_{1}=0.025 \mathrm{~m}, l_{2}=0.025 \mathrm{~m}$, and $l_{3}=0.0125 \mathrm{~m}$. The Hiroshima-hand grasps two cylindrical objects whose radial length is $r=0.01 \mathrm{~m}$. Since we did not measure the position of the center of gravity of the objects, we calculated it by using their kinematic relationships.

We considered manipulating the objects without causing relative motion among them. The grasp satisfies the second kind of AFC, and such manipulation is possible. We used the following simple controller for the control of object motion:

$$
\boldsymbol{\tau}=\mathbf{K}\left(\mathbf{D}_{C} \mathbf{J}\right)^{T} \mathbf{D}_{B} \mathbf{D}_{G}\left[\begin{array}{c}
\mathbf{p}_{G d}-\mathbf{p}_{G} \\
-\theta_{G}
\end{array}\right]+\boldsymbol{\tau}_{\text {bias }}
$$

where $\mathbf{K}, \tau_{\text {bias }}$, and $\theta_{G}$ denote the gain matrix, the bias torque, and the rotation angle of the center of mass of the two objects, respectively, and where we set $\mathbf{K}=\operatorname{diag}\left[\begin{array}{lll}20 & 20 & 20\end{array}\right] \mathrm{mNm} / \mathrm{deg}$. The desired trajectory is set in such a way that the center of mass moves on the left side for $0.02 \mathrm{~m}$ in $13 \mathrm{~s}$ and moves back to the original position in $13 \mathrm{~s}$. We can see from Figure 7 that the objects follow the desired trajectory well

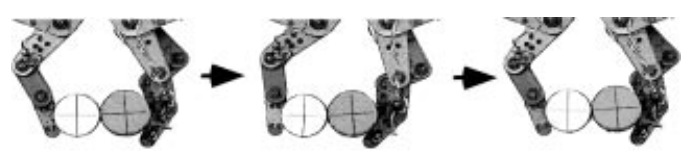

(a) Motion of objects

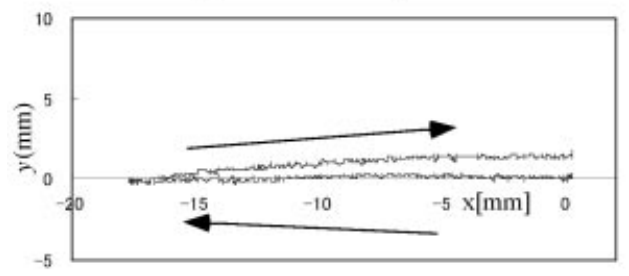

(b) Trajectory of left-hand object

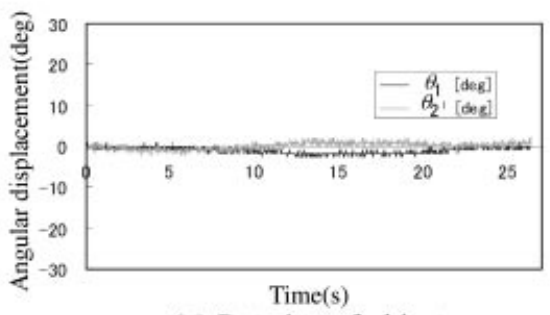

(c) Rotation of objects

Figure 7. Experimental results
(Fig. 7(b)), and that relative motion among the objects does not occur (Fig. 7(c)), since the rotation angles of the two objects are almost the same.

\section{DISCUSSION}

In Section 6, we considered three kinds of planar grasp of three objects (Fig. 3). Among them, case 2 satisfies the first kind of AFC, and the acceleration can be generated at the center of gravity of one of the objects in an arbitrary direction. However, even if an arbitrary acceleration can be generated on one of the objects, the direction of acceleration generated on the other objects is unknown. Therefore, as soon as the acceleration is exerted on the object, the grasp configuration will change, as shown in case 1 . Since case 1 does not satisfy the first kind of AFC, the grasp no longer satisfies the first kind of AFC. Therefore, for a grasp satisfying the first kind of AFC, arbitrary manipulation is impossible.

If the grasp satisfies the second kind of AFC, the objects can be manipulated like a single rigid body. On the other hand, the number of fingers required for the second kind of AFC $(n>(3 m-2 r) / 2)$ is usually greater than that for the first kind of AFC $(n \geq 3)$. Therefore, if there are multiple objects to be manipulated, we should first consider manipulating them using the second kind of AFC. If there are not enough fingers for the second kind of AFC, then we should try to manipulate the objects using the first kind of AFC. Using the first kind of AFC, we can move at least one object in the desired direction.

As an example of grasp planning for the second kind of AFC, case 3 of Figure 3 is considered. We can calculate that the necessary number of fingers is four. To construct the second kind of AFC, we first focus on one of the objects, especially the one in the center. If the object in the center is grasped by two fingers, it satisfies AFC for a single object, since the necessary number of fingers for a single object is two. Now the system composed of two fingers and the object in the center can be regarded as a finger (region A in Fig. 8). Then we focus on the object on the right-hand side. Since the object on the right-hand side is in contact with region $\mathrm{A}$, the object satisfies $\mathrm{AFC}$ if there is one more finger in contact with the object. Now, the system composed of region $\mathrm{A}$, the object on the right-hand side, and a finger, can be regarded as a finger (region $B$ in Fig. 8). Finally, we focus on the object on the lefthand side. Since the object on the left-hand side is in contact with region $\mathrm{B}$, the object satisfies $\mathrm{AFC}$ if there is one more finger in contact with the object. Hence we 


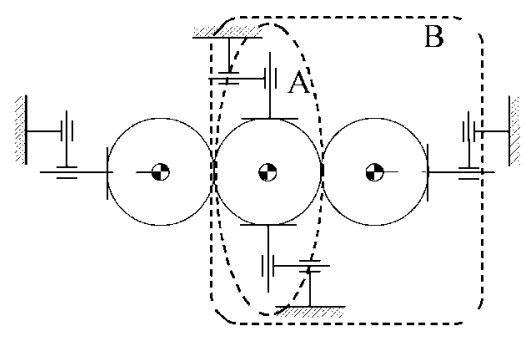

Figure 8. Construction of second kind of AFC.

can confirm that each object satisfies AFC for a single object and that the second kind of AFC for multiple objects can be satisfied.

\section{CONCLUSIONS}

This article has discussed active force closure (AFC) for multiple objects. We provided the relationship between the force at the center of gravity of the objects and the contact force. Without taking the relative motion into account, we defined the first kind of AFC as being focused on each object, and the second kind of AFC as being focused on the center of mass of the grasp. The numerical examples and experiments for general 3D cases will be our future research topic.

\section{ACKNOWLEDGMENTS}

We would like to express our sincere gratitude to Tatsuya Shirai of Suzuka Technical College and Koji Furutera with Sharp Co. Ltd., for their help with the experiments and the numerical examples.

\section{REFERENCES}

1. K. Harada and M. Kaneko, Enveloping grasp for multiple objects. Proc 1998 IEEE Int Conf on Robotics and Automation, 1998, pp. 2409-2415.

2. K. Harada, M. Kaneko, and T. Tsuji, Rolling based manipulation for multiple objects. IEEE Trans Robot Automat 16:(5) (2000), 457-468.

3. J.K. Salisbury and B. Roth, Kinematics and force analysis of articulated hands. ASME J Mech Trans Automat Des 105 (1982), 33-41.

4. J.C. Trinkle, On the stability and instantaneous velocity of grasped frictionless objects. IEEE Trans Robot Automat 8:(5) (1992), 560-572.

5. T. Yoshikawa, Passive and active closures by constraining mechanisms. Proc 1996 IEEE Int Conf on Robotics and Automation, 1996, pp. 1477-1484.
6. P. Dauchez and X. Delebarre, Force-controlled assembly of two objects with a two-arm robot. Robotica 9 (1991), 299-306.

7. K. Kosuge, M. Sakai, and K. Kanitani, Decentralized coordinated motion control of manipulators with vision and force sensors. Proc 1995 IEEE Int Conf on Robotics and Automation, 1995, pp. 2456-2462.

8. Y. Aiyama, M. Minami, and T. Arai, Manipulation of multiple objects by two manipulators. Proc 1998 IEEE Int Conf on Robotics and Automation, 1998, pp. 29042909.

9. R. Mattikalli, D. Baraff, P. Khosla, and B. Repetto, Gravitational stability of frictionless assemblies. IEEE Trans Robot Automat 11:(3) (1995), 374-388.

10. F. Reuleaux, The kinematics of machinery. Macmillan, New York, 1876.

11. M.S. Ohwovoriole, An extension of screw theory and its application to the automation of industrial assemblies. Ph.D. dissertation, Department of MechanicalEngineering, Stanford University, 1980.

12. B. Mishra, J.T. Schwartz, and M. Sharir, On the existence and synthesis of multifinger positive grips. Algorithmica (Special Issue: Robotics) 2:(4) (1987), 541-558.

13. V. Nguyen, Constructing force closure grasps. Int J Robot Res 7:(3) (1988), 3-16.

14. Y.C. Park and G.P. Starr, Grasp synthesis of polygonal objects using a three-fingered robot hand, Int J Robot Res 11:(3) (1992), 163-184.

15. I.-M. Chen and J.W. Burdick, A qualitative test fornfinger force-closure grasps on planar objects with applications to manipulation and finger gaits. Proc 1993 IEEE Int Conf on Robotics and Automation, 1993, pp. 814-820.

16. J. Ponce and B. Faverjon, On computing three-finger force-closure grasps of polygonal objects. IEEE Trans Robot Automat 11:(6) (1995), 868-881.

17. J. Kerr and B. Roth, Analysis of multifingered hands. Int J Robot Res 4:(4) (1986), 3-17.

18. Y. Nakamura, K. Nagai, and T. Yoshikawa, Dynamics and stability in coordination of multiple robotic mechanisms. Int J Robot Res 8:(2) (1989), 44-61.

19. C. Ferrari and J. Canny, Planning optimal grasps. Proc 1992 IEEE Int Conf on Robotics and Automation, 1992, pp. 2290-2295.

20. J.-C. Latombe, Robot motion planning. Kluwer, Boston 1991.

21. R.M. Murray, Z. Li, and S.S. Sastry, A mathematical introduction to robotic manipulation. CRC Press, Florida 1994.

22. J. Pertin-Troccaz, Grasping: A state of art. O. Khatib, J. Craig, and T. Lozano-Perez (Eds.), In: The Robotic review, Cambridge, MA: MIT Press, 1989.

23. A. Bicchi, On the closure properties of robotic grasping. Int J Robot Res 14:(4) (1995), 319-334.

24. X.-Z. Zhang, Y. Nakamura, K. Goda, and K. Yoshimoto. Robustness of power grasp. Proc 1994 IEEE Int Conf on Robotics and Automation, 1994, pp. 2828-2835.

25. Y. Yu, K. Takeuchi, and T. Yoshikawa, Optimization of robot hand power grasps. Proc 1998 IEEE Int Conf on Robotics and Automation, 1998, pp. 3341-3347.

26. N. Imamura, M. Kaneko, and T. Tsuji, Development of three-fingered robot hand with a new design concept. Proc IASTED Int Conf Robotics and Manufacturing, 1998, pp. 44-49, Banff, Canada. 